\title{
On Possible A-Priori “Imprinting” of General Relativity Itself on the Performed Lense-Thirring Tests with LAGEOS Satellites
}

\author{
Lorenzo Iorio \\ INFN-Sezione di Pisa Permanent address for correspondence, Viale Unità di Italia, Bari (BA), Italy \\ E-mail: lorenzo.iorio@libero.it
}

Received October 23, 2009; accepted December 22, 2009

\begin{abstract}
The impact of possible a-priori "imprinting" effects of general relativity itself on recent attempts to measure the general relativistic Lense-Thirring effect with the LAGEOS satellites orbiting the Earth and the terrestrial geopotential models from the dedicated mission GRACE is investigated. It is analytically shown that general relativity, not explicitly solved for in the GRACE-based models, may "imprint" their even zonal harmonic coefficients of low degrees $J_{\ell}$ at a non-negligible level, given the present-day accuracy in recovering them. This translates into a bias of the LAGEOS-based relativistic tests as large as the Lense-Thirring effect itself. Further analyses should include general relativity itself in the GRACE data processing by explicitly solving for it.
\end{abstract}

Keywords: experimental studies of gravity, satellite orbits, harmonics of the gravity potential field

\section{Introduction}

The term "gravitomagnetism" [1-3] (GM) denotes those gravitational phenomena concerning orbiting test particles, precessing gyroscopes, moving clocks and atoms and propagating electromagnetic waves $[4,5]$ which, in the framework of the Einstein's General Theory of Relativity (GTR), arise from non-static distributions of matter and energy. In the weak-field and slow motion approximation, the Einstein field equations of GTR, which is a highly non-linear Lorentz-covariant tensor theory of gravitation, get linearized [6], thus looking like the Maxwellian equations of electromagntism. As a consequence, a "gravitomagnetic" field $\vec{B}_{g}$, induced by the off-diagonal components $g_{0 i}, i=1,2,3$ of the space-time metric tensor related to mass-energy currents, arises. In particular, far from a localized slowly rotating body with angular momentum $\vec{S}$ the gravitomagnetic field can be written as [7]

$$
\vec{B}_{g}(\vec{r})=\frac{G}{c r^{3}}[\vec{S}-3(\vec{S} \cdot \hat{r}) \hat{r}],
$$

where $G$ is the Newtonian gravitational constant and $c$ is the speed of light in vacuum. It affects, e.g., a test particle moving with velocity $v$ with a non-central acceleration [7]

$$
\vec{A}_{\mathrm{GM}}=\left(\frac{\vec{v}}{c}\right) \times \vec{B}_{g} .
$$

It is the cause of the so-called Lense-Thirring ${ }^{1}$ effect [9], which is one of the most famous and empirically inves-

\footnotetext{
${ }^{1}$ According to a recent historical analysis, it should be more correct to
} speak about an Einstein-Thirring-Lense effect [8] tigated GM features; another one is the gyroscope precession [10,11], goal of the Gravity Probe B (GP-B) mission [12] whose data analysis is still ongoing [13].

The Lense-Thirring effect consists of small secular precessions of the longitude of the ascending node $\Omega$ and the argument of pericenter $\omega$ of the orbit of a test particle in geodesic motion around a slowly rotating body with angular momentum $\vec{S}$; they are

$$
\dot{\Omega}_{\mathrm{LT}}=\frac{2 G S}{c^{2} a^{3}\left(1-e^{2}\right)^{3 / 2}}, \quad \dot{\omega}_{\mathrm{LT}}=-\frac{6 G S \cos I}{c^{2} a^{3}\left(1-e^{2}\right)^{3 / 2}}
$$

where $a$ is the semimajor axis of the satellite's orbit, $e$ is its eccentricity and $I$ is the inclination of the orbital plane to the equatorial plane of the central body.

Concerning the possibilities of measuring it in the terrestrial gravitational field, soon after the dawn of the space age with the launch of Sputnik in 1957 it was proposed by Soviet scientists to directly test the LenseThirring effect with artificial satellites orbiting the Earth. In particular, V. L. Ginzburg [14-16] proposed to use the perigee of a terrestrial spacecraft in highly elliptic orbit, while A. F. Bogorodskii [17] considered also the node. In 1977-1978 Cugusi and Proverbio [18,19] suggested to use the passive geodetic satellite LAGEOS, in orbit around the Earth since 1976 and tracked with the Satellite Laser Ranging (SLR) technique, along with the other existing laser-ranged targets to measure the LenseThirring node precession. Since such earlier studies it was known that a major source of systematic error is represented by the fact that the even $(\ell=2,4,6, \ldots)$ zonal $(m=$ 
0) harmonic coefficients $J_{\ell}, \ell=2,4,6$ of the multipolar expansion of the classical part of the terrestrial gravitational potential, accounting for its departures from spherical symmetry due to the Earth's diurnal rotation, induce competing secular precessions of the node and the perigee of satellites [20] whose nominal sizes are several orders of magnitude larger than the Lense-Thirring ones. In the case of the node, the largest precession is due to the first even zonal harmonic $J_{2}$

$$
\dot{\Omega}_{J_{2}}=-\frac{3}{2} n\left(\frac{R_{\oplus}}{a}\right) \frac{\cos I J_{2}}{\left(1-e^{2}\right)^{2}},
$$

where $R_{\oplus}$ is the Earth's mean equatorial radius and $n$ $=\sqrt{G M_{\oplus} / a^{3}}$ is the satellite's Keplerian mean motion. For the other higher degrees the analytical expressions are more involved; since they have already been published in e.g., [21], we will not show them here.

Tests have started to be effectively performed about 15 years ago by Ciufolini and coworkers [22] with the LAGEOS and LAGEOS II satellites ${ }^{2}$, according to a strategy by Ciufolini [23] involving the use of a suitable linear combination of the nodes $\Omega$ of both satellites and the perigee $\omega$ of LAGEOS II in order to remove the impact of the first two multipoles of the non-spherical gravitational potential of the Earth. Latest tests have been reported by Ciufolini and Pavlis [24,25], Lucchesi [26] and Ries and coworkers [27] with only the nodes of both the satellites according to a combination of them explicitly proposed by Iorio $^{3}$ [28]. The total uncertainty reached is still matter of debate [32-38] because of the lingering uncertainties in the Earth's multipoles and in how to evaluate their biasing impact; it may be as large as $\sim 20-30 \%$ according to conservative evaluations [32,35-38], while more optimistic views $[24,25,27]$ point towards $\sim 10-15 \%$.

To be more specific, the node-only combination used in the latest tests is

$$
\dot{\Omega}^{\mathrm{LAGEOS}}+k_{1} \dot{\Omega}^{\mathrm{LAGEOS} \mathrm{II}}, k_{1}=0.554 \text {. }
$$

It was designed to remove the effects of the static and time-varying components of $J_{2}$, so that (5) is affected by the remaining even zonals of higher degree $J_{4}, J_{6}, \ldots$ The gravitomagnetic trend predicted by (5) amounts to 47.8 milliarcseconds year ${ }^{-1}$ (mas yr ${ }^{-1}$ in the following) since the Lense-Thirring node precessions for the LAGEOS satel-

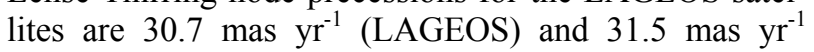
(LAGEOS II). The Lense-Thirring signal is usually extracted from long time series of computed "residuals" of the nodes of LAGEOS and LAGEOS II obtained by processing their data with a suite of dynamical force

\footnotetext{
${ }^{2}$ LAGEOS II was launched in 1992.

${ }^{3}$ See also [29-31].

${ }^{4}$ Actually, the nodes are not directly measurable quantities, so that speaking of "residuals" is somewhat improper.

${ }^{5}$ For a critical discussion of such an issue, see [41].

${ }^{6} \mathrm{See}$ on the WEB http://icgem.gfz-potsdam.de/ICGEM/ICGEM.html.
}

models which purposely do not encompass the gravitomagnetic force itself $[39,40]$. The action of the even zonals is accounted for by using global solutions for the Earth's gravity field, in which general relativity has never been explicitly solved for ${ }^{5}$, produced by several institutions around the world from data of dedicated satellite-based missions like GRACE ${ }^{6}$ [42].

GRACE recovers the spherical harmonic coefficients of the geopotential from the tracking of both satellites by GPS and from the observed intersatellite distance variations [43]. The possible "memory" effect of the gravitomagnetic force in the satellite-to-satellite tracking was preliminarily addressed in [32]. Here we will focus on the "imprint" which may come from the GRACE orbits which is important for us because it mainly resides in the low degree even zonals.

\section{A-Priori "Imprinting" of General Relativity on the GRACE-Based Models}

Concerning that issue, Ciufolini and Pavlis write in [33] that such a kind of leakage of the Lense-Thirring signal itself into the even zonals retrieved by GRACE is completely negligible because the GRACE satellites move along (almost) polar orbits. Indeed, for perfectly polar (I $=90 \mathrm{deg})$ trajectories, the gravitomagnetic force is entirely out-of-plane, while the perturbing action of the even zonals is confined to the orbital plane itself. According to Ciufolini and Pavlis [33], the deviations of the orbit of GRACE from the ideal polar orbital configuration would have negligible consequences on the "imprint" issue. In particular, they write: "the values of the even zonal harmonics determined by the GRACE orbital perturbations are substantially independent on the a priori value of the Lense-Thirring effect [...]. The small deviation from a polar orbit of the GRACE satellite, that is $1.7 \times 10^{-2} \mathrm{rad}$, gives only rise, at most, to a very small correlation with a factor $1.7 \times 10^{-2} \%$. The meaning of such a statement is unclear; anyway, we will show below that such a conclusion is incorrect.

The relevant orbital parameters of GRACE are quoted in Table 1; the orbital plane of GRACE is, in fact, shifted by $0.98 \mathrm{deg}$ from the ideal polar configuration, and contrary to what claimed in [33], this does matter because its classical secular node precessions are far from being negligible with respect to our issue. The impact of the Earth's gravitomagnetic force on the even zonals retrieved by GRACE can be quantitatively evaluated by computing the "effective" value $\bar{C}_{\ell 0}^{\mathrm{LT}}$ of the normalized even zonal gravity coefficients which would induce classical secular node precessions for GRACE as large as those due to its Lense-Thirring effect, which is independent of the inclination $I$. To be more precise, $\bar{C}_{\ell 0}^{\mathrm{LT}}$ come from solving the following equation which connects the classical even zonal ${ }^{7}$ It must be recalled that $J_{\ell}=-\sqrt{2 \ell+1} \bar{C}_{\ell 0}$ where $\bar{C}_{\ell 0}$ are the normalized gravity coefficients. 
precession of degree $\ell \dot{\Omega}_{J_{\ell}}=\dot{\Omega}_{\ell} J_{\ell}$ to the Lense-Thirring node precession $\dot{\Omega}_{\mathrm{LT}}$

$$
\dot{\Omega}_{\ell} J_{\ell}=\dot{\Omega}_{\mathrm{LT}}
$$

In it

$$
\dot{\Omega}_{\ell}=f\left(a, e, I ; R_{\oplus}, G M_{\oplus}\right)
$$

are the coefficients of the classical node precessions depending on the satellite's orbital parameters and on the Earth's radius and mass. Table 2 lists $\bar{C}_{\ell 0}^{\mathrm{LT}}$ for degrees $\ell=4,6$, which are the most effective in affecting the combination (5). Thus, the gravitomagnetic field of the Earth contributes to the value of the second even zonal of the geopotential retrieved from the orbital motions of GRACE by an amount of the order of $2 \times 10^{-10}$, while for $\ell=6$ the imprint is one order of magnitude smaller. Given the present-day level of accuracy of the latest GRACEbased solutions, which is of the order of $10^{-12}$ (Table 3), effects as large as those of Table 2 cannot be neglected. Thus, we conclude that the influence of the Earth's gravitomagnetic field on the low-degree even zonal harmonics of the global gravity solutions from GRACE may exist, falling well within the present-day level of measurability.

\section{The Impact of the "Imprint" on the LAGEOS-LAGEOS II Tests}

A further, crucial step consists of evaluating the impact of such an a-priori "imprint" on the test conducted with the LAGEOS satellites and the combination of Equation (5): if the LAGEOS-LAGEOS II uncancelled combined classical geopotential precession computed with the GRACEbased a-priori "imprinted" even zonals of Table 2 is a relevant part of, or if it is even larger than the combined Lense-Thirring precession, it will be demonstrated that the doubts concerning the a-priori gravitomagnetic "memory" effect are founded. It turns out that this is just the case because Equation (5) and Table 2 yield a combined geopotential precession whose magnitude is 77.8

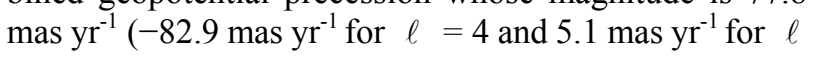
$=6$ ), i.e. just 1.6 times the Lense-Thirring signal itself. This means that the part of the LAGEOS-LAGEOS II uncancelled classical combined node precessions which is affected by the "imprinting" by the Lense-Thirring force through the GRACE-based geopotential's spherical harmonics is as large as the LAGEOS-LAGEOS II combined gravitomagnetic signal itself.

We, now, comment on how Ciufolini and Pavlis reach a different conclusion. They write in [33]: "However, the Lense-Thirring effect depends on the third power of the inverse of the distance from the central body, i.e., $(1 / r)^{3}$, and the $J_{2}, J_{4}, J_{6} \ldots$ effects depend on the powers $(1 / r)^{3.5},(1 / r)^{5.5},(1 / r)^{7.5} \ldots$ of the distance; then, since the ratio of the semimajor axes of the GRACE satellites to the
Table 1. Orbital parameters of GRACE and its Lense-Thirring node precession. Variations of the orders of about 10 $\mathbf{k m}$ in the semimajor axis $a$ and $0.001 \mathrm{deg}$ in the inclination $I$ may occur, but it turns out that they are irrelevant in our discussion. (http://www.csr.utexas.edu/grace/ground/ globe.html)

\begin{tabular}{cccc}
\hline$a(\mathrm{~km})$ & $e$ & $I(\mathrm{deg})$ & $\dot{\Omega}_{\mathrm{LT}}\left(\mathrm{mas} \mathrm{yr}^{-1}\right)$ \\
\hline 6835 & 0.001 & 89.02 & 177.4 \\
\hline
\end{tabular}

Table 2. Effective "gravitomagnetic" normalized gravity coefficients for GRACE ( $\ell=4,6 ; m=0)$. They have been obtained by comparing the GRACE classical node precessions to the Lense-Thirring rate. Thus, they may be viewed as a quantitative measure of the leakage of the LenseThirring effect itself into the second and third even zonal harmonics of the global gravity solutions from GRACE. Compare them with the much smaller calibrated errors in $\bar{C}_{40}$ and $\bar{C}_{60}$ of the GGM03S model [44] of Table 3

\begin{tabular}{cc}
\hline $\bar{C}_{40}^{\mathrm{LT}}$ & $\bar{C}_{60}^{\mathrm{LT}}$ \\
\hline $2.23 \times 10^{-10}$ & $-2.3 \times 10^{-11}$ \\
\hline
\end{tabular}

Table 3. Calibrated errors in the solved-for normalized gravity coefficients $\bar{C}_{40}$ and $\bar{C}_{60}$ according to the GGM03S global gravity solution by CSR [44]. They can be publicly retrieved at http:// icgem.gfz-potsdam.de/ ICGEM/ ICGEM. html. Compare them with the much larger "gravitomagnetic” imprinted coefficients of Table 2

\begin{tabular}{cc}
\hline$\sigma \bar{C}_{40}$ & $\sigma \bar{C}_{60}$ \\
\hline $4 \times 10^{-12}$ & $2 \times 10^{-12}$ \\
\hline
\end{tabular}

LAGEOS' satellites is $\sim \frac{6780}{12270} \approx 1.8$, any conceivable "LenseThirring Imprint" on the spherical harmonics at the GRACE altitude becomes quickly, with increasing distance, a negligible effect, especially for higher harmonics of degree $\ell>4$. Therefore, any conceivable "LenseThirring imprint" is negligible at the LAGEOS' satellites altitude." From such statements it seems that they compare the classical GRACE precessions to the gravitomagnetic LAGEOS' ones. This is meaningless since, as we have shown, one has, first, to compare the classical and relativistic precessions of GRACE itself, with which the Earth's gravity field is solved for, and, then, compute the impact of the relativistically "imprinted" part of the GRACE-based even zonals on the combined LAGEOS nodes. These two stages have to be kept separate, with the first one which is fundamental; if different satellite(s) $\mathrm{Y}$ were to be used to measure the gravitomagnetic field of the Earth, the impact of the Lense-Thirring effect itself on them should be evaluated by using the "imprinted" even zonals evaluated in the first stage. Finally, in their latest statement Ciufolini and Pavlis write in [33]: "In addition, in (Ciufolini et al. 1997), it was proved with several 
simulations that by far the largest part of this "imprint" effect is absorbed in the by far largest coefficient $J_{2}$." Also such a statement, in the present context, has no validity since the cited work refers to a pre-GRACE era. Moreover, no quantitative details at all were explicitly released concerning the quoted simulations, so that it is not possible to judge by.

\section{Conclusions}

We have analytically investigated the impact of possible a-priori "imprinting" effects of GTR itself on the ongoing Lense-Thirring tests with the LAGEOS satellites in the gravitational field of the Earth modeled from the dedicated GRACE mission.

The classical part of the terrestrial gravitational potential, acting as a source of major systematic error because of its even zonal harmonic coefficients $\bar{C}_{\ell 0}$, is retrieved from the data of the dedicated satellite-based GRACE mission. GTR, not explicitly solved for so far in GRACE data analyses, may impact the retrieved even zonals of the GRACE models at a non-negligible level $\left(\approx 10^{-10}-10^{-11}\right.$ for $\ell=4,6$ ), given the present-day level of accuracy (for $\ell=$ $4,6)$. It turns out that the resulting a-priori "imprint" of the Lense-Thirring effect itself on the LAGEOS-LAGEOS II data analysis performed to test it is of the same order of magnitude of the general relativistic signal itself.

Further, more robust tests should rely upon Earth gravity models in which GTR is explicitly solved for.

\section{REFERENCES}

[1] K. S. Thorne, "Gravitomagnetism, jets in quasars, and the stanford gyroscope experiment," in Near Zero: New Frontiers of Physics, J. D. Fairbank, B. S. Deaver, C. W. F. Everitt, and P. F. Michelson, Eds., W. H. Freeman and Company, New York, pp. 573-586, 1988.

[2] W. Rindler, Relativity. Special, General and Cosmological, Oxford University Press, Oxford, pp. 195-198, 2001.

[3] B. Mashhoon, "Gravitoelectromagnetism: a brief review", in The Measurement of Gravitomagnetism: A Challenging Enterprise, L. Iorio, Ed., Nova, Hauppauge, pp. 29-39, 2007.

[4] M. L. Ruggiero and A. Tartaglia, "Gravitomagnetic effects," II Nuovo Cimento B, Vol. 117, No. 7, pp. 743768, July 2002.

[5] G. Schäfer, "Gravitomagnetic effects," General Relativity and Gravitation, Vol. 36, No. 10, pp. 2223-2235, October 2004.

[6] H. C. Ohanian and R. J. Ruffini, Gravitation and Spacetime, 2nd Edition, W. W. Norton and Company, New York, pp. 130-240, 1994.

[7] B. Mashhoon, L. Iorio, and H. I. M. Lichtenegger, "On the gravitomagnetic clock effect," Physics Letters A, Vol.
292, No. 1-2, pp. 49-57, December 2001.

[8] H. Pfister, "On the history of the socalled Lense-Thirring effect," General Relativity and Gravitation, Vol. 39, No. 11, pp. 1735-1748, November 2007.

[9] J. Lense and H. Thirring, "Über den Einfluß der Eigenrotation der Zentralkörper auf die Bewegung der Planeten und Monde nach der Einsteinschen Gravitationstheorie," Physikalische Zeitschrift, Vol. 19, pp. 156-163, 1918.

[10] G. E. Pugh, Proposal for a satellite test of the Coriolis prediction of general relativity WSEG Research Memorandum, The Pentagon, Washington DC, No. 11, November 1959.

[11] L. I. Schiff, "Possible new experimental test of general relativity theory," Physical Review Letters, Vol. 4, No. 5, pp. 215-217, March 1960.

[12] C. W. F. Everitt, S. Buchman, D. B. DeBra, G. M. Keiser, J. M. Lockhart, B. Muhlfelder, B. W. Parkinson, J. P. Turneaure and other members of the Gravity Probe B team, "Gravity Probe B: Countdown to Launch," in Gyros, Clocks, Interferometers: Testing Relativistic Gravity in Space, C. Lämmerzahl, C. W. F. Everitt and F. W. Hehl, Eds., Springer, Berlin, pp. 52-82, 2001.

[13] C. W. F. Everitt, M. Adams, W. Bencze, S. Buchman, B. Clarke, J. W. Conklin, D. B. DeBra, M. Dolphin, M. Heifetz, D. Hipkins, T. Holmes, G. M. Keiser, J. Kolodziejczak, J. Li, J. Lipa, J. M. Lockhart, J. C. Mester, B. Muhlfelder, Y. Ohshima, B. W. Parkinson, M. Salomon, A. Silbergleit, V. Solomonik, K. Stahl, M. Taber, J. P. Turneaure, S. Wang, and P. W. Worden, "Gravity Probe B data analysis," Space Science Reviews, Vol. 148, No. 1-4, pp. 53-69, December 2009.

[14] V. L. Ginzburg, "The use of artificial earth satellites for verifying the general theory of relativity," Advances in Physical Science (Uspekhi Fizicheskikh Nauk), Vol. 63, No. 1, pp. 119-122, 1957.

[15] V. L. Ginzburg, "Artificial satellites and the theory of relativity," Scientific American, Vol. 200, No. 5, pp. 149160, May 1959.

[16] V. L. Ginzburg, "Experimental verifications of the general theory of relativity," in Recent Developments in General Relativity, Pergamon press, London, pp. 57-71, 1962.

[17] A. F. Bogorodskii, "Relativistic effects in the motion of an artificial earth satellite," Soviet Astronomy, Vol. 3, No. 5, pp. 857-862, October 1959.

[18] L. Cugusi and E. Proverbio, "Relativistic effects on the motion of the earth's satellites," Journal of Geodesy, Vol. 51, pp. 249-252, 1977.

[19] L. Cugusi and E. Proverbio, "Relativistic effects on the motion of earth's artificial satellites," Astronomy and Astrophysics, Vol. 69, pp. 321-325, October 1978.

[20] W. M. Kaula, Theory of Satellite Geodesy, Blaisdell, Waltham, 1966.

[21] L. Iorio, "The impact of the static part of the Earth's gravity field on some tests of General Relativity with satellite laser ranging," Celestial Mechanics and Dynamical 
Astronomy, Vol. 86, No. 3, pp. 277-294, July 2003.

[22] I. Ciufolini, D. M. Lucchesi, F. Vespe, and A. Mandiello, "Measurement of dragging of inertial frames and gravitomagnetic field using laser-ranged satellites," II Nuovo Cimento A, Vol. 109, No. 5, pp. 575-590, May 1996.

[23] I. Ciufolini, "On a new method to measure the gravitomagnetic field using two orbiting satellites," II Nuovo Cimento A, Vol. 109, No. 12, pp. 1709-1720, December 1996.

[24] I. Ciufolini and E. C. Pavlis, "A confirmation of the general relativistic prediction of the Lense-Thirring effect," Nature, Vol. 431, No. 7011, pp. 958-960, October 2004.

[25] I. Ciufolini, E. C. Pavlis and R. Peron, "Determination of frame-dragging using Earth gravity models from CHAMP and GRACE," New Astronomy, Vol. 11, No. 8, pp. 527550, July 2006.

[26] D. M. Lucchesi, "The lense thirring effect measurement and LAGEOS satellites orbit analysis with the new gravity field model from the CHAMP mission," Advances in Space Research, Vol. 39, No. 2, pp. 324-332, 2007.

[27] J. C. Ries, R. J. Eanes and M. M. Watkins, "Confirming the frame-dragging effect with satellite laser ranging," in Proceedings of The 16th International Laser Ranging Workshop, "SLR-The Next Generation", Poznań (PL), 13-17 October 2008, S. Schillak, Ed. Available from: http://cddis.gsfc.nasa.gov/lw16/.

[28] L. Iorio, "The new Earth gravity models and the measurement of the lense-thirring effect," in The Tenth Marcel Grossmann Meeting On Recent Developments in Theoretical and Experimental General Relativity, Gravitation and Relativistic Field Theories. Proceedings of the MG10 Meeting, Rio de Janeiro, Brazil 20-26 July 2003, M. Novello, S. P. Bergliaffa, and R. J. Ruffini, Eds. Singapore: World Scientific, 2006, pp. 1011-1020.

[29] E. C. Pavlis, "Geodetic contributions to gravitational experi- ments in space," in Recent Developments in General Relativity: Proceedings of the 14th SIGRAV Conference on General Relativity and Gravitational Physics (Genova, IT, 18-22 September 2000), R. Cianci, R. Collina, M. Francaviglia, and P. Fré P., Eds. Milan: Springer, 2002, pp. 217-233.

[30] J. C. Ries, R. J. Eanes and B. D. Tapley, "Lense-thirring precession determination from laser ranging to artificial satellites," in Nonlinear Gravitodynamics, The LenseThirring Effect, R. J. Ruffini and C. Sigismondi, Eds., World Scientific, Singapore, pp. 201-211, 2003.

[31] J. C. Ries, R. J. Eanes, B. D. Tapley, and G. E. Peterson, "Prospects for an improved lense-thirring test with slr and the GRACE gravity mission," in Proceedings of The 13th International Laser Ranging Workshop, NASA CP (2003-212248), R. Noomen, S. Klosko, C. Noll and M. Pearlman, Eds., NASA Goddard, Greenbelt, 2003, $\mathrm{http}: / /$ cddisa.gsfc.nasa.gov/lw13/lw_proceedings.html\#science.

[32] L. Iorio, "On the reliability of the so-far performed tests for measuring the Lense-Thirring effect with the LAG
EOS satellites," New Astronomy, Vol. 10, No. 8, pp. 603-615, August 2005.

[33] I. Ciufolini and E. C. Pavlis, "On the measurement of the Lense-Thirring effect using the nodes of the LAGEOS satellites, in reply to 'On the reliability of the so-far performed tests for measuring the Lense-Thirring effect with the LAGEOS satellites' by L. Iorio," New Astronomy, Vol. 10, No. 8, pp. 636-651, August 2005.

[34] D. M. Lucchesi, "The impact of the even zonal harmonics secular variations on the lense-thirring effect measurement with the two lageos satellites," International Journal of Modern Physics D, Vol. 14, No. 12, pp. 1989-2023, 2005.

[35] L. Iorio, "A critical analysis of a recent test of the lense-thirring effect with the LAGEOS satellites," Journal of Geodesy, Vol. 80, No. 3, pp. 128-136, June 2006.

[36] L. Iorio, "An assessment of the measurement of the lense-thirring effect in the earth gravity field, in reply to: "On the measurement of the lense-thirring effect using the nodes of the LAGEOS satellites, in reply to "On the reliability of the sofar performed tests for measuring the lense-thirring effect with the LAGEOS satellites" by L. Iorio," by I. Ciufolini and E. Pavlis," Planetary and Space Science, Vol. 55, No. 4, pp. 503-511, March 2007.

[37] L. Iorio, "An assessment of the systematic uncertainty in present and future tests of the lense-thirring effect with satellite laser ranging," Space Science Reviews, Vol. 148, No. 1-4, pp. 363-381, December 2009.

[38] L. Iorio, "Conservative evaluation of the uncertainty in the LAGEOS-LAGEOS II lense-thirring test," Central European Journal of Physics, Vol. 8, No. 1, pp. 25-32, February 2010.

[39] D. M. Lucchesi and G. Balmino, "The LAGEOS satellites orbital residuals determination and the lense thirring effect measurement," Planetary and Space Science, Vol. 54, No. 6, pp. 581-593, May 2006.

[40] D. M. Lucchesi, "The LAGEOS satellites orbital residuals determination and the way to extract gravitational and non-gravitational unmodeled perturbing effects," Advances in Space Research, Vol. 39, No. 10, pp. 15591575, 2007.

[41] K. Nordtvedt jr., "Slr contributions to fundamental physics," Surveys in Geophysics, Vol. 22, No. 5-6, pp. 597602, September 2001.

[42] B. D. Tapley and Ch. Reigber, "The GRACE mission: status and future plans," EOS Transactions AGU 2001; 82: Fall Meeting Supplement G41, C-02.

[43] C. Reigber, R. Schmidt, F. Flechtner, R. König, U. Meyer, K. H. Neumayer, P. Schwintzer and S. Y. Zhu, "An earth gravity field model complete to degree and order 150 from GRACE: EIGEN-GRACE02S," Journal of Geodynamics, Vol. 39, No. 1, pp. 1-10, January 2005.

[44] B. D. Tapley, J. C. Ries, S. Bettadpur, D. Chambers, M. Cheng, F. Condi and S. Poole, "The GGM03 mean earth gravity model from GRACE," American Geophysical Union, Fall Meeting, abstract \# G42A-03, 2007. 